\title{
The feasibility and technical strategy of a fascia space priority approach in laparoscopic lateral lymph node dissection for advanced middle and low rectal cancer: a retrospective multicentre study
}

\author{
Yi Sun ${ }^{1}$, Lei Lian², Hong Zhang ${ }^{3}$, Xuefeng Bai ${ }^{4}$, Zhongshi Xie ${ }^{5}$, Jun Ouyang ${ }^{6}$, Kai Wang7, Hang Yuan ${ }^{8}$, Chang Xu ${ }^{9}$, \\ Henggui Luo ${ }^{10}$, Haijun Deng ${ }^{11}$, Jun $\mathrm{Li}^{12}$, Hongjie Yang ${ }^{1}$, Zhichun Zhang ${ }^{1}$, Peng Li ${ }^{1}$, Xipeng Zhang ${ }^{1}$ \\ ${ }^{1}$ Department of Colorectal Surgery, Tianjin Union Medical Centre, Tianjin, China \\ ${ }^{2}$ Department of Colorectal Surgery, the Sixth Affiliated Hospital of Sun Yat-sen University, Guangzhou, China; Guangdong Institute \\ of Gastroenterology, Guangdong Provincial Key Laboratory of Colorectal and Pelvic Floor Diseases, the Sixth Affiliated Hospital \\ of Sun Yat-sen University, Guangzhou, China \\ ${ }^{3}$ Department of Colorectal Surgery, Shengjing hospital of China Medical University, Shenyang, China \\ ${ }^{4}$ Department of Colorectal Surgery, Affiliated Tumour Hospital of Harbin Medical University, Harbin, China \\ ${ }^{5}$ Department of Gastrointestinal Colorectal Surgery, China-Japan Union Hospital of Jilin University, Changchun, China \\ ${ }^{6}$ Department of Gastrointestinal Surgery, The First Affiliated Hospital of University of South China, Hengyang, Hunan, China \\ ${ }^{7}$ Department of Gastrointestinal Surgery, The Affiliated Hospital of Xuzhou Medical University, Xuzhou, China \\ ${ }^{8}$ The Surgical Department of Coloproctology, Zhejiang Provincial People's Hospital, People's Hospital of Hangzhou Medical College, \\ Hangzhou, China \\ ${ }^{9}$ Department of Colorectal Surgery, The First Affiliated Hospital of Wenzhou Medical University, Xuzhou, China \\ ${ }^{10}$ The First Department of General Surgery, Xiangtan Central Hospital, Xiangtan, China \\ ${ }^{11}$ Department of General Surgery, Nanfang Hospital, Southern Medical University, Guangzhou, China \\ ${ }^{12}$ Department of Colorectal Surgery and Oncology, Key Laboratory of Cancer Prevention and Intervention, Ministry of Education, \\ The Second Affiliated Hospital, Zhejiang University School of Medicine, Hangzhou, China
}

\begin{abstract}
Introduction: Laparoscopic lateral lymph node dissection (LLND) is an important treatment for patients with lateral lymph node metastasis.

Aim: To assess the technical feasibility and investigate the surgical outcomes after LLND using the fascia space priority approach for patients with advanced middle and low rectal cancer.

Material and methods: Consecutive patients undergoing laparoscopic LLND using the fascia space priority approach from June 2017 to June 2020 were identified from 12 medical centres in mainland China. Three anatomic fascia spaces were dissected to establish the boundaries of the LLND, and the obturator and internal iliac lymph nodes were excised in an en bloc manner. Retrospective clinical data including patient characteristics, surgical details, and pathology were analysed.

Results: A total of 112 patients were identified. All surgeries were completed laparoscopically with no conversions. The mean operation time was $343.6 \pm 103.8 \mathrm{~min}$ for the entire procedure. The median blood loss was $100 \mathrm{ml}$ (range: 100-700 ml). The median lymph node yield was 6 (range: 1-41), and lymph nodes were positive in 39.3\% (44/112)
\end{abstract}

Address for correspondence

Xipeng Zhang, Department of Colorectal Surgery, Tianjin Union Medical Centre, 300121 Tianjin, China, e-mail: zhangxipeng@vip.tom.com 
of the patients. Sixteen (14.3\%) patients had Clavien-Dindo I-II complications, no Clavien-Dindo III-IV complications were identified. The incidence of complications between the bilateral dissection group and the unilateral dissection group was not statistically different $(p=0.19)$. The complication rate between the " $C R T$ " group and the "no nCRT" group was not significantly different $(p=0.62)$ either. There were no perioperative deaths.

Conclusions: Laparoscopic LLND using the fascia space priority approach is feasible and safe for patients with lateral lymph node metastasis.

Key words: lateral lymph node dissection, rectal cancer, fascia space priority approach, surgical technology.

\section{Introduction}

With the widespread implementation of neoadjuvant chemoradiotherapy ( $\mathrm{nCRT}$ ) and total mesorectal excision (TME), the local recurrence rate of rectal cancer has decreased to $5 \%$ to $10 \%$, and recurrence in the lateral lymph nodes has become one of the crucial factors in local recurrence [1]. The integrity of the TME specimen is a key determinant in assessing the safety of laparoscopic rectal cancer surgery [2, 3]. Lateral lymph node metastasis is an important route of lymph node spread in rectal cancer. Neither lateral lymph node dissection (LLND) alone nor nCRT plus TME can completely prevent metastasis and recurrence in the lateral lymph nodes [4-6], but LLND after nCRT in patients with suspected lateral lymph node metastasis has been associated with reduced local recurrence [7].

However, LLND is a far more complex procedure than TME alone, especially after nCRT. The difficulty of the operation and the risk of surgical complications may be greatly increased after nCRT because of tissue oedema, fibrosis, and enlarged lymph nodes invading the iliac vessels [8]. Konishi carried out lateral lymph node dissection using fascia guidance and pushed forward this technology [7]. Despite the awareness of fascia guidance in this technique consume surgery, most surgeons in China still hesitate to perform it. Therefore, we developed the technology of a fascia space priority approach for LLND. First, the anatomical boundaries were established by using the fascia space, and then the LLND was carried out. We have previously reported this technique for laparoscopic LLND, which can standardise the procedure [9]. To promote the application of this technique, we have conducted a variety of training courses in China, including surgical video presentations and didactic lectures in anatomy. As a result, surgeons at more and more medical centres are mastering and successfully applying the fascia space priority approach for laparoscopic LLND.
In this retrospective multicentre study, we aimed to investigate the technical feasibility and safety of laparoscopic LLND using the fascia space priority approach for patients with advanced middle and low rectal cancer.

\section{Aim}

This study aimed to assess the technical feasibility and investigate the surgical outcomes after LLND using the fascia space priority approach for patients with advanced middle and low rectal cancer.

\section{Material and methods}

\section{Centres}

A total of 12 centres across mainland China which have adopted the fascia space priority approach for laparoscopic LLND participated in this study. All the centres involved in this study are high-volume centres with more than 200 operations performed for low rectal cancer each year. All of the operating surgeons from these centres received training in the technique and each centre was equipped with a high-resolution laparoscopic imaging system. The participating centres are listed in Figure 1.

\section{Case selection}

Medical records of consecutive patients undergoing laparoscopic LLND from participating centres across mainland China from June 2017 to June 2020 were reviewed. Inclusion criteria were as follows: (i) Diagnosis of advanced middle or low rectal adenocarcinoma (inferior tumour border below the peritoneal reflection) confirmed by colonoscopy with biopsy. (ii) For patients who received preoperative CRT, the short-axis diameters of lateral lymph nodes were greater than $7 \mathrm{~mm}$ on magnetic resonance imaging (MRI) before nCRT and greater than $5 \mathrm{~mm} \mathrm{MRI} \mathrm{after}$ nCRT. (iii) For patients without preoperative CRT, the short-axis diameters of lateral lymph nodes were at 


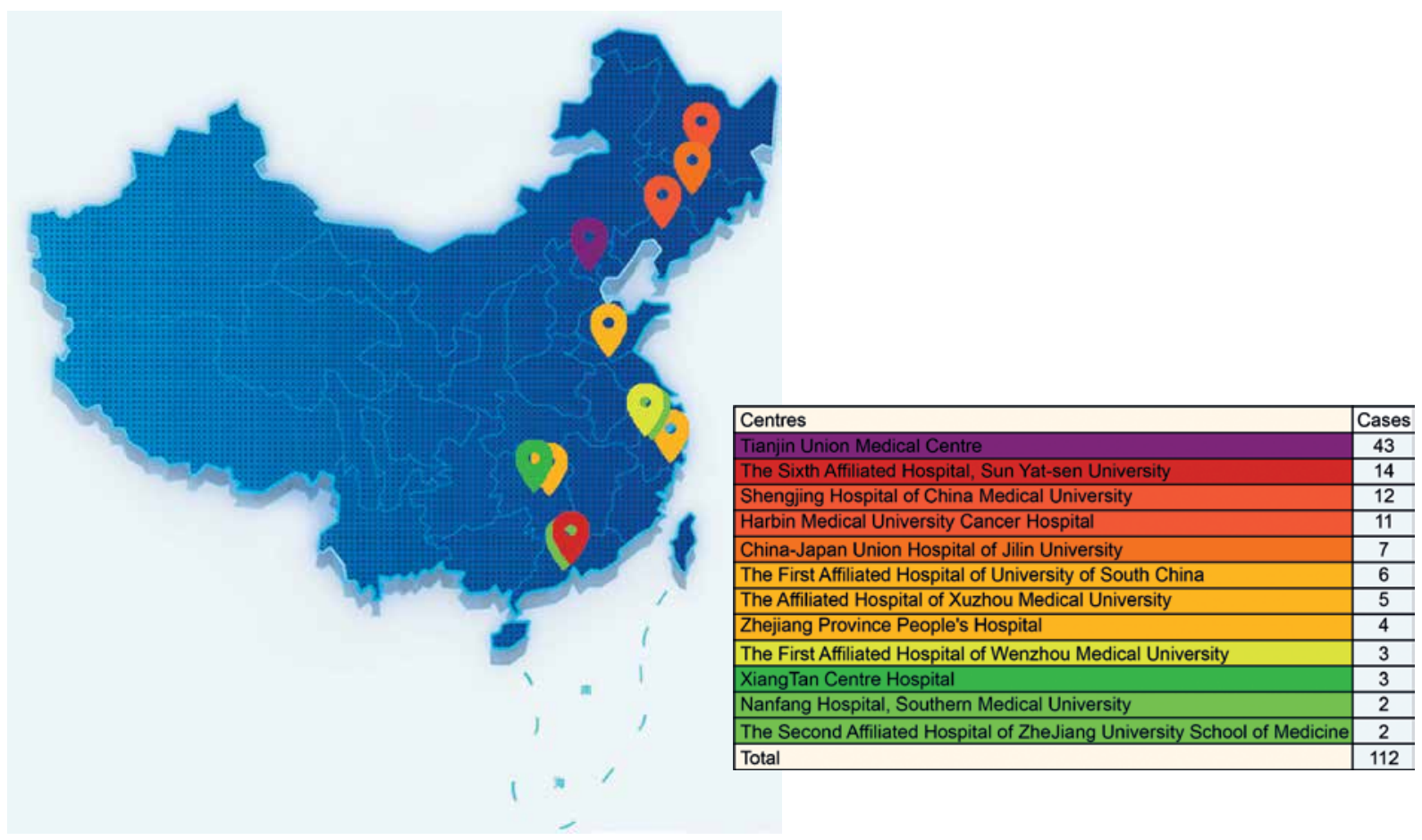

Figure 1. Medical centres and number of cases

least $7 \mathrm{~mm}$ on preoperative staging MRI. Exclusion criteria were: (i) Unresectable distant metastasis. (ii) Tumour invasion requiring combined visceral organ resection.

We recommended nCRT when the short diameter of the largest lymph node was at least $7 \mathrm{~mm}$ on pre-treatment staging MRI. If, after 5 weeks of $\mathrm{nCRT}$, the short diameter of the largest lymph node was at least $5 \mathrm{~mm}$ on the preoperative MRI, TME + LLND was performed 6 to 8 weeks after nCRT. If the preoperative short diameter of the largest lymph node was less than $5 \mathrm{~mm}$, follow-up observation was recommended. TME + LLND was also performed in patients without $\mathrm{nCRT}$ when the radial resection margin was negative.

\section{Surgical procedure}

LLND was unilateral or bilateral depending on the presence of suspected lymph node metastasis, and it was performed following TME. The internal iliac lymph nodes and the obturator lymph nodes were defined as the major fields of dissection. All procedures were performed using the fascia space priority approach, as shown in our published video [9].

The procedure is commenced by establishing 3 anatomic fascia spaces, including the space between the ureterohypogastric nerve fascia and the medial aspect of the vesicohypogastric fascia, the space between the vesicohypogastric fascia and the obturator lymph nodes, and the space between the obturator lymph nodes and the parietal pelvic fascia (Photo 1). The obturator lymph nodes and the internal iliac lymph nodes are then dissected and excised en bloc. Finally, communication between the LLND and TME compartments is established (Photo 2). The third anatomic fascia space lateral to the external iliac vessels is dissected to enter the space between the parietal pelvic fascia and the obturator lymph nodes but the group 293 lymph nodes are retained. Adequate dissection of the 3 anatomic fascia spaces and fully revealing the internal pudendal artery (IPA) is mandatory during this procedure. All of the videos were obtained and reviewed by the same surgical team.

\section{Clinical parameters and patient follow-up}

An electronic data collection sheet was sent to participating centres and patient profiles, surgical data, pathological data, and short-term prognosis were collected and analysed. Patients had follow-up visits every 3 months during the first year after the surgery and every 6 months thereafter until 5 years after the operation. 

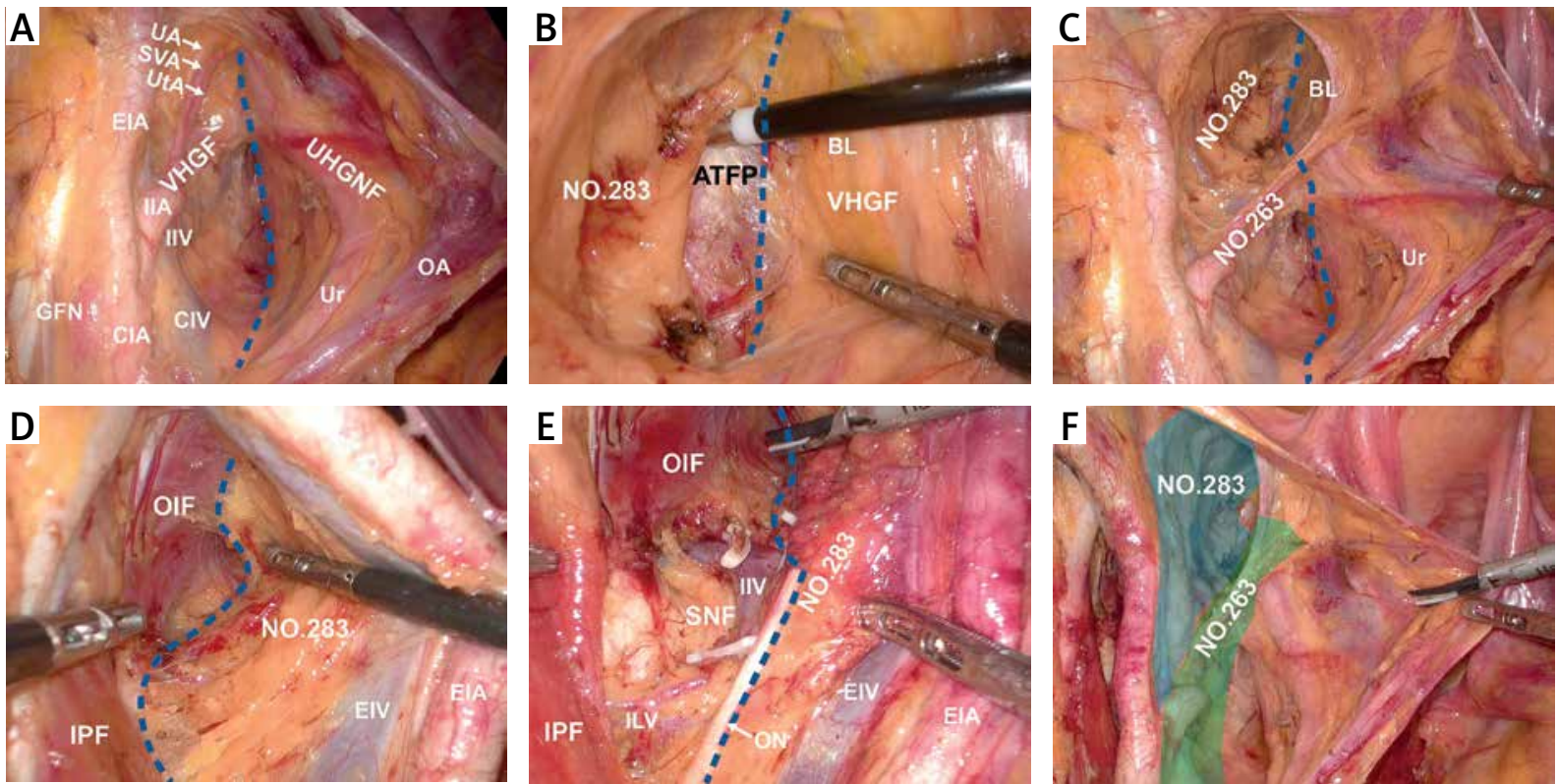

Photo 1. Establishment of 3 anatomic fascia spaces. A - Intraoperative view after dissection of the first anatomic fascia space between the ureterohypogastric nerve fascia and the medial aspect of the vesicohypogastric fascia. B - Intraoperative view after dissection of the second anatomic fascia space between the vesicohypogastric fascia and the obturator lymph nodes. NO 283, number 283 (obturator region) lymph nodes; ATFP, arch of the tendinous pelvic fascia. C - View of the first and the second anatomic fascia spaces. NO 263, number 263 (internal iliac region) lymph nodes. D - Intraoperative view after dissection of the third anatomic fascia space between the parietal pelvic fascia and the obturator lymph nodes. $\mathbf{E}-\mathrm{View}$ of the dorsal wall. $\mathbf{F}$ - The locations of the lateral lymph node groups [9]

UA - umbilical artery, SVA - superior vesical artery, UtA - uterine artery, EIA - external iliac artery, VHGF - vesicohypogastric fascia, IIA - internal iliac artery, IIV - internal iliac vein, UHGNF - ureterohypogastric nerve fascia, GFN - genitofemoral nerve, CIA - common iliac artery, CIV - common iliac vein, Ur - ureter, OA - ovarian vessels, OIF - obturator internus fascia, IPF - iliopsoas fascia, EIV - external iliac vein, EIA - external iliac artery, SNF- sciatic nerve fascia, ON - obturator nerve.
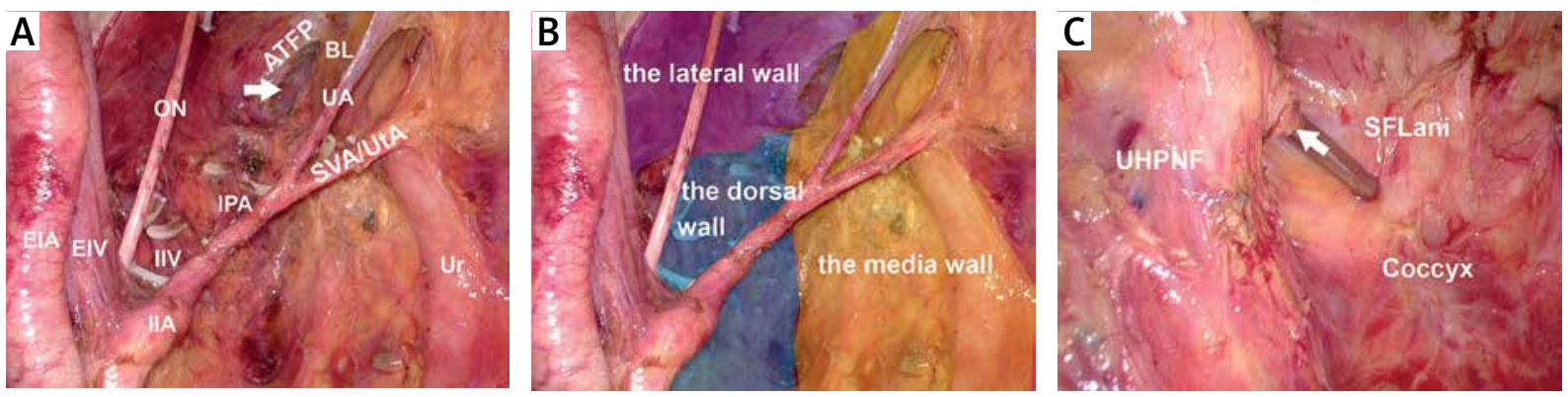

Photo 2. Intraoperative view after lateral lymph node dissection. A - The surgical field after dissection of the lateral lymph nodes. B - View of the lateral, dorsal, and medial walls. $\mathbf{C}$ - Communication between the LLND and TME compartments [9]

$B L$ - bladder, IPA - iliopsoas muscle, UHPNF - ureterohypogastric nerve fascia, SFLani-superior fascia of the levator ani. 
Table I. Patient characteristics $(n=112)$

\begin{tabular}{|c|c|}
\hline Variable & Value \\
\hline Age [years] $\bar{x} \pm s$ & $56 \pm 13.1$ \\
\hline \multicolumn{2}{|l|}{ Sex, $n(\%):$} \\
\hline Male & $68(60.7)$ \\
\hline Female & $44(39.3)$ \\
\hline $\mathrm{BMI}\left[\mathrm{kg} / \mathrm{m}^{2}\right] \bar{x} \pm s$ & $24.1 \pm 3.2$ \\
\hline Distance to tumour from AV $[\mathrm{cm}]$ mean (range) & $4.5(0-10.0)$ \\
\hline \multicolumn{2}{|l|}{ Preoperative clinical stage: } \\
\hline 1 & $1(0.9)$ \\
\hline ॥ & $23(20.5)$ \\
\hline III & $88(78.6)$ \\
\hline History of abdominal operation, $n(\%)$ & $20(17.9)$ \\
\hline $\mathrm{nCRT}, n(\%)$ & $58(49.1)$ \\
\hline
\end{tabular}

Table II. Surgical outcomes $(n=112)$

\begin{tabular}{|lc|}
\hline Variable & Value \\
\hline Operation time $[\mathrm{min}] \bar{x} \pm s$ & $343.6 \pm 103.8$ \\
\hline Blood loss [ml] mean (range) & $100(10-700)$ \\
\hline $\begin{array}{l}\text { Post-operative hospital stay [days] median } \\
\text { (range) }\end{array}$ & $13(4-34)$ \\
\hline \begin{tabular}{l} 
Lateral lymph node dissection, $n(\%):$ \\
\hline Unilateral
\end{tabular} & $74(66.1)$ \\
\hline Bilateral & $38(33.9)$ \\
\hline Conversion, $n(\%)$ & $0(0)$ \\
\hline
\end{tabular}

Table III. Post-operative complications $(n=112)$

\begin{tabular}{|c|c|}
\hline Variable & Value \\
\hline \multicolumn{2}{|l|}{ Perioperative complications, $n(\%)$ : } \\
\hline Postoperative haemorrhage & $0(0)$ \\
\hline Lymphatic leak & $3(2.7)$ \\
\hline Lymphatic cyst & $4(3.6)$ \\
\hline Urine leak & $0(0)$ \\
\hline Long-term urinary retention ( $\geq 3$ months) & $0(0)$ \\
\hline Anastomotic leakage & $4(3.6)$ \\
\hline Intestinal obstruction & $4(3.6)$ \\
\hline Lower extremity oedema & $3(2.7)$ \\
\hline Lower limb pain & $0(0)$ \\
\hline Lower limb mobility disorder & $0(0)$ \\
\hline Total complications rate, $n(\%)$ & $16(14.3)$ \\
\hline Perioperative mortality, $n$ (\%) & 0 \\
\hline Re-operation rate, $n(\%)$ & 0 \\
\hline
\end{tabular}

\section{Ethical approval}

All human studies were reviewed by the appropriate ethics committee and have therefore been performed in accordance with the ethical standards laid down in an appropriate version of the 1965 Declaration of Helsinki.

\section{Statistical analysis}

Data were analysed using SPSS Statistics (Version 25.0). The normally distributed numerical variables are expressed as the mean \pm SD. The statistical significance of differences was evaluated by Student's $t$-test. The non-normal distributed numerical variables are expressed as the median (range). The statistical significance of differences was evaluated by Mann-Whitney $U t$-test. Categorical variables are reported as frequencies and percentages and were compared using the $\chi^{2}$ test. A $p$-value $<0.05$ was considered significant.

\section{Results}

\section{Patient characteristics}

A total of 112 patients were included, and their characteristics are summarised in Table I. There were 44 men and 68 women. The mean age was 56 \pm 13.1 years, and the mean body mass index (BMI) was $24.1 \pm 3.2 \mathrm{~kg} / \mathrm{m}^{2}$. The mean distance from the distal edge of the tumour to the anal verge was 4.5 (range: $0-10.0) \mathrm{cm}$. Most of the patients (78.6\%; 88 cases) had clinical stage III adenocarcinomas.

\section{Surgical parameters and complications}

The surgical parameters are summarised in Table II. Unilateral and bilateral LLND was performed in $74(66.1 \%)$ and 38 (33.9\%) patients, respectively. The operation time was recorded from skin discission to incision closure. The median operation time was $343.6 \pm 103.8 \mathrm{~min}$. The median blood loss was $100 \mathrm{ml}$ (range: 100-700 ml). The median post-operative length of hospital stay was 13 (range: 4-34) days. Sixteen patients had complications (14.3\%), but there were no perioperative deaths. All the complications were grade I and II according to the Clavien-Dindo classification. And there were no grade III and IV complications (Table III). All patients consented to laparoscopic TME + LLND, and there were no conversions to laparotomy. The clinical data between the "nCRT" group and the "no nCRT" group are compared in Table IV, and the data show that there are no significant dif- 
Table IV. Clinical data of nCRT and no nCRT

\begin{tabular}{|c|c|c|c|}
\hline Variable & $\mathrm{nCRT}(n=55)$ & No nCRT $(n=57)$ & $P$-value \\
\hline Age [years] $\bar{x} \pm s$ & $51.5 \pm 12.4$ & $61.1 \pm 10.9$ & $<0.001$ \\
\hline \multicolumn{4}{|l|}{ Sex, $n(\%):$} \\
\hline Male & $32(58.2)$ & $36(63.2)$ & 0.70 \\
\hline Female & $23(41.8)$ & $21(36.8)$ & \\
\hline $\mathrm{BMI}\left[\mathrm{kg} / \mathrm{m}^{2}\right] \bar{x} \pm s$ & $23.7 \pm 2.9$ & $24.0 \pm 3.5$ & 0.61 \\
\hline Operation time $[\mathrm{min}] \bar{x} \pm s$ & $352.2 \pm 110.7$ & $335.3 \pm 97.0$ & 0.40 \\
\hline Blood loss [ml] mean (range) & $100(20-700)$ & $100(10-500)$ & 0.90 \\
\hline Post-operative hospital stay [days] median (range) & $13.5(4-34)$ & $12(7-33)$ & 0.58 \\
\hline \multicolumn{4}{|l|}{ Lateral lymph node dissection, $n$ (\%): } \\
\hline Unilateral & $34(61.8)$ & $40(70.2)$ & 0.43 \\
\hline Bilateral & $21(38.2)$ & $17(29.8)$ & \\
\hline Number of patients with positive lateral lymph nodes, $n(\%)$ & $21(38.2)$ & $23(40.4)$ & 0.85 \\
\hline Complication rate (Clavien-Dindo I, II), n (\%) & $8(14.5)$ & $11(19.3)$ & 0.62 \\
\hline Complication rate (Clavien-Dindo III, IV), n (\%) & $0(0)$ & $0(0)$ & \\
\hline
\end{tabular}

ferences between the 2 groups in terms of operative time, intraoperative blood loss, and incidence of complications. The clinical data of the "unilateral" group and the "bilateral" group is illustrated in Table V. Significant differences are observed in the operative time $(p<0.001)$ and the overall intraoperative blood loss $(p=0.01)$. The complication rate between the "nCRT" group and the "no nCRT" group shows no significant different $(p=0.62)$ either.

\section{Post-operative pathology}

The pathology findings are summarised in Table VI. Patients with pathological stage III tumours constituted the largest group ( $n=66$ patients; $58.9 \%$ ). The median lymph node yield was 6 (range: $1-41)$, and lymph nodes were positive in $39.3 \%$ (44/112) of the patients.

\section{Discussion}

Nowadays, the standard treatment for locally advanced lower rectal cancer without enlarged lateral lymph nodes is neoadjuvant chemoradiation therapy (NACRT) followed by TME, and the local recurrence rate under standard treatment has decreased to $5 \%$ to $10 \%$. However, it is reported that lateral lymph node metastasis is present in $16 \%$ to $23 \%$ of

Table V. Clinical data of unilateral group and bilateral group

\begin{tabular}{|c|c|c|c|}
\hline Variable & Unilateral $(n=74)$ & Bilateral $(n=38)$ & $P$-value \\
\hline Age [years] $\bar{x} \pm s$ & $57.0 \pm 13.1$ & $55.3 \pm 11.7$ & 0.51 \\
\hline \multicolumn{4}{|l|}{ Sex, $n(\%):$} \\
\hline Male & $48(64.9)$ & $20(52.6)$ & 0.23 \\
\hline Female & $26(35.1)$ & $18(47.4)$ & \\
\hline $\mathrm{BMI}\left[\mathrm{kg} / \mathrm{m}^{2}\right] \bar{x} \pm \mathrm{s}$ & $23.9 \pm 3.3$ & $23.6 \pm 3.2$ & 0.64 \\
\hline Operation time $[\mathrm{min}] \bar{x} \pm s$ & $316.8 \pm 89.4$ & $395.1 \pm 111.2$ & 0.00 \\
\hline Blood loss [ml] mean (range) & $100(10-700)$ & $200(20-700)$ & 0.01 \\
\hline Post-operative hospital stay [days] median (range) & $12(4-33)$ & $13.5(7-34)$ & 0.07 \\
\hline $\mathrm{nCRT}, n(\%)$ & $34(45.9)$ & $21(55.3)$ & 0.43 \\
\hline Number of patients with positive lateral lymph nodes, $n(\%)$ & $21(38.2)$ & $23(40.4)$ & 0.85 \\
\hline Complications rate (Clavien-Dindo I, II), n (\%) & $10(13.5)$ & $9(23.7)$ & 0.19 \\
\hline Complications rate (Clavien-Dindo III, IV), n (\%) & $0(0)$ & $0(0)$ & \\
\hline
\end{tabular}


Table VI. Clinicopathological characteristics $(n=112)$

\begin{tabular}{|lc|}
\hline Variable & Value \\
\hline TNM stage, $n(\%):$ & \\
\hline 0 & $4(3.6)$ \\
\hline II & $7(6.2)$ \\
\hline Number of lymph nodes per each side & $35(31.3)$ \\
\hline $\begin{array}{l}\text { Number of patients with positive lateral } \\
\text { lymph nodes, } n(\%)\end{array}$ & $66(58.9)$ \\
\hline
\end{tabular}

advanced lower rectal cancer cases, if LLND is not performed, and the local recurrence rate is as high as $20 \%$ [10]. Therefore, LLND is an important procedure for locally advanced lower rectal cancer with clinically suspected swollen lymph nodes. Laparoscopy is a popular technique in colorectal surgery around the world due to its remarkable advantages [11] However, it is well known that laparoscopic LLND in cases of advanced middle and low rectal cancer with enlarged lateral lymph nodes is a complex and challenging surgery. Especially for patients receiving nCRT, a variety of factors such as tissue oedema, fibrosis, and enlarged lymph nodes invading the iliac vessels can greatly increase the difficulty of LLND [8]. In spite of these difficulties, our results showed that all 112 laparoscopic LLND operations were successfully completed. We attribute this success to the fascia space priority approach. The priority is the dissection of the avascular fascia space to establish the lymph node boundary so that the surgical anatomy is precise and the field of vision is clean, and thus the surgical risk and difficulty are significantly reduced. Meanwhile, bleeding and exudation caused by incision into lymphatic tissues are avoided, and incidental injuries caused by inadequate haemostasis are minimised.

There were $16(14.3 \%)$ patients with complications. Early in the study period, 4 patients developed lymphocytic cysts, which can be caused by incomplete drainage of lymph fluid in the area of the lateral lymph nodes. We improved the technique by cutting the tendinous arch of the pelvic fascia to create communication between the LLND and TME compartments and allow the drainage fluid to be completely discharged. Consequently, the rate of lymphocytic cysts decreased sharply. Another 3 pa- tients developed unilateral lower extremity oedema after surgery. After excluding the possibility of deep vein thrombosis, the oedema was thought to be caused by lower extremity lymphatic obstruction after dissection of the external iliac lymph nodes. We addressed this by avoiding dissection of external iliac lymph nodes when there were no suspected clinical metastases in the lymph nodes surrounding the external iliac vessels on the preoperative MRI, and the occurrence rate of lower extremity oedema caused by lymphatic obstruction was reduced.

There are other potentially serious complications of LLND that can severely limit quality of life. Ureteral and bladder injury leads to urinary leakage, pelvic autonomic nerve injury leads to dysuria [12], and sciatic nerve injury leads to lower limb movement disorders and pain. However, there were no severe surgical complications in our study. By giving priority to the dissection of 3 non-vascularised fascia spaces, the fascia space priority approach ensures that the lateral lymph nodes are isolated, and the ureter, pelvic autonomic nerves, bladder, neurovascular bundle, and sciatic nerve are separated and protected, thereby reducing the risk of serious damage to these vital structures. Thus, there was no patient with long-term urinary retention ( $\geq 3$ months), urinary fistula, lower limb pain, or mobility disorder in our group.

The average operation time was $343.6 \mathrm{~min}$, which showed no obvious advantage over previous studies $[8,13]$. This might be related to the number of cases (33.9\%; 38/112 patients) of bilateral lymph node metastasis in our study. The difficulty of surgery increased because of tissue oedema and fibrosis after neoadjuvant, we compared the data between patients underwent neoadjuvant therapy and those who not, the data shows no significant difference in term of surgical time, blood loss and incidence of complications, indicating that the patents after nCRT are also suitable for LLND using fascia space priority approach. The incidence of complications between the bilateral dissection group and unilateral dissection group was not statistically different $(p=0.19)$, and the autonomic nerve fascia was well maintained as an intact nerve layer may contribute to it.

Our results showed that up to $39.3 \%$ of patients had positive lateral lymph nodes postoperatively, higher than other related studies [8], which relates to the strict selection of surgical indications and surgical quality control. In order to thoroughly dissect the lymph nodes around the internal pudendal ar- 
tery, which are most at risk of lateral lymph node metastasis, the internal pudendal artery is exposed up to the level of the pudendal canal or removed if it is invaded by metastatic lymph nodes [14]. After CRT, tissue fibrosis or invasion by enlarged lymph nodes often causes adhesions between the distal branches of the internal iliac vessels and the enlarged lymph nodes. Under such circumstances, we adopted the strategy of resecting both the lymph nodes and the fused vessels to dissect the lymph nodes radically. For bilateral LLND cases, if the superior vesical artery is not invaded, it should be preserved to secure the blood supply to the bladder.

Dr. Liang contends that laparoscopic LLND after CRT is so technically challenging that not every surgeon can perform it [15]. The fascia space priority approach we have developed can standardise LLND. However, for surgeons who are not familiar with the technique, we recommend systematic training, including equipped courses, as suggested by Perez [16]. Moreover, as advised in the newly developed transanal (ta) TME, an anatomy lesson, which can shorten the preliminary learning curve and decrease the risk of perioperative complications, can also be taken into consideration [17]. All of the centres in our study had at least one systematic training, including theoretical instruction, fresh frozen cadaver anatomy, and on-site surgical demonstrations.

The fascia space priority approach can make uniform the process of laparoscopic LLND. We have promoted this technique by conducting anatomy and surgery training courses, and trained surgeons have mastered the technique in a short time. During the study period, there were 2 centres performing only 2 cases after the training courses.

Nevertheless, there are some limitations to this research: First, this was a retrospective study, sexual function was not evaluated, urological function was evaluated only on the basis of the time of catheter removal, and no scale evaluation was performed. We are currently conducting a prospective study of urinary function. Second, the number of cases carried out in some centres was small due to the short period of adopting this procedure. Although their surgery met the standard of the fascia space priority approach, there was still some deviation in the separation technology, operation time, and blood lost. Finally, from the oncology perspective, the follow-up time is relatively short.

\section{Conclusions}

Laparoscopic LLND using a fascia space priority approach is feasible for patients with advanced middle and low rectal cancer. The successful application of this technique in 12 Chinese medical centres proves that it is safe and feasible. Multi-perspective and prospective randomised controlled trials and outcomes of long-term oncology follow-up will be needed to confirm the therapeutic efficacy.

\section{Acknowledgments}

Tianjin Health Science and Technology Project (ZC20081).

\section{Conflict of interest}

The authors declare no conflict of interest.

\section{References}

1. Baik SH, Kim NK, Lee YC, et al. Prognostic significance of circumferential resection margin following total mesorectal excision and adjuvant chemoradiotherapy in patients with rectal cancer. Ann Surg Oncol 2007; 14: 462-9.

2. Wang R, Wei Z, Liu Q, et al. Transanal versus transabdominal specimen extraction in laparoscopic rectal cancer surgery: a retrospective analysis from China. Videosurgery Miniinv 2019; 14: 203-9

3. Ferko A, Orhalmi J, Dusek T, et al. Higher risk of incomplete mesorectal excision and positive circumferential margin in low rectal cancer regardless of surgical technique. Videosurgery Miniinv 2014; 9: 569-77.

4. Ogura A, Konishi T, Cunningham C, et al. Neoadjuvant (chemo) radiotherapy with total mesorectal excision only is not sufficient to prevent lateral local recurrence in enlarged nodes: results of the multicenter lateral node study of patients with low CT3/4 rectal cancer. J Clin Oncol 2019; 37: 33-43.

5. Aiba T, Uehara K, Mukai T, et al. Transanal extended rectal surgery with lateral pelvic lymph node dissection. Tech Coloproctol 2018; 22: 893-94.

6. Ishihara S, Kawai K, Tanaka T, et al. Diagnostic value of FDG$\mathrm{PET} / \mathrm{CT}$ for lateral pelvic lymph node metastasis in rectal cancer treated with preoperative chemoradiotherapy. Tech Coloproctol 2018; 22: 347-54.

7. Nakanishi R, Yamaguchi T, Akiyoshi T, et al. Laparoscopic and robotic lateral lymph node dissection for rectal cancer. Surg Today 2020; 50: 209-16.

8. Yang X, Gu C, Hu T, et al. Is laparoscopic selective lateral lymph node dissection for locally advanced rectal cancer after neoadjuvant chemoradiotherapy safe? ANZ J Surg 2019; 89: E492-7.

9. Sun Y, Zhang Z, Zhou Y, Zhang X. Fascial space priority approach in laparoscopy: lateral pelvic lymph node dissection for advanced low rectal cancer. Tech Coloproctol 2020; 24: 335-6.

10. Watanabe J, Ishibe A, Suwa Y, et al. Short- and long-term outcomes of laparoscopic versus open lateral lymph node dis- 
Yi Sun, Lei Lian, Hong Zhang, Xuefeng Bai, Zhongshi Xie, Jun Ouyang, Kai Wang, Hang Yuan, Chang Xu, Henggui Luo, Haijun Deng, Jun Li, Hongjie Yang, Zhichun Zhang, Peng Li, Xipeng Zhang

section for locally advanced middle/lower rectal cancer using a propensity score-matched analysis. Surg Endosc 2020 Sep 1. doi: 10.1007/s00464-020-07943-4.

11. Böyük A, Aday U, Gültürk B, et al. Effect of splenic flexure mobilization performed via medial-to-lateral and superior-to-inferior approach on early clinical outcomes in elective laparoscopic resection of rectal cancer. Videosurgery Miniinv 2019; 14: 509-15.

12. Maeda K, Maruta M, Utsumi T, et al. Bladder and male sexual functions after autonomic nerve-sparing TME with or without lateral node dissection for rectal cancer. Tech Coloproctol 2003; 7: 29-33.

13. Konishi T, Kuroyanagi H, Oya M, et al. Lateral lymph node dissection with preoperative chemoradiation for locally advanced lower rectal cancer through a laparoscopic approach. Surg Endosc 2011; 25: 2358-9.

14. Ueno $\mathrm{H}$, Mochizuki $\mathrm{H}$, Hashiguchi Y, et al. Potential prognostic benefit of lateral pelvic node dissection for rectal cancer located below the peritoneal reflection. Ann Surg 2007; 245: 80-7.

15. Liang JT. Technical feasibility of laparoscopic lateral pelvic lymph node dissection for patients with low rectal cancer after concurrent chemoradiation therapy. Ann Surg Oncol 2011; 18: 153-9.

16. Perez RO, São Julião GP, Vailati BB, et al. Lateral node dissection in rectal cancer in the era of minimally invasive surgery: a stepby-step description for the surgeon unacquainted with this complex procedure with the use of the laparoscopic approach. Dis Colon Rectum 2018; 61: 1237-40.

17. Francis N, Penna M, Mackenzie H, et al.; International TaTME Educational Collaborative Group. Consensus on structured training curriculum for transanal total mesorectal excision (TaTME). Surg Endosc 2017; 31: 2711-9.

Received: 22.02.2021, accepted: 3.03.3021 